\title{
Heteroepitaxy between wurtzite and corundum materials
}

$\operatorname{AUTHOR}(S)$ :

Hayashi, Yuki; Banal, Ryan G.; Funato, Mitsuru; Kawakami, Yoichi

\section{CITATION:}

Hayashi, Yuki ... [et al]. Heteroepitaxy between wurtzite and corundum materials. Journal of Applied Physics 2013, 113(18): 183523.

\section{ISSUE DATE:}

2013-05-13

URL:

http://hdl.handle.net/2433/187965

\section{RIGHT:}

(c) 2013 American Institute of Physics. This article may be downloaded for personal use only. Any other use requires prior permission of the author and the American Institute of Physics. 


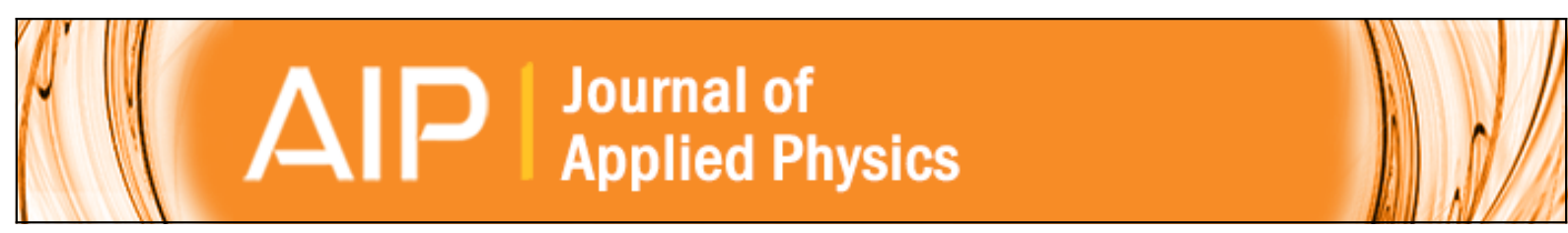

\section{Heteroepitaxy between wurtzite and corundum materials}

Yuki Hayashi, Ryan G. Banal, Mitsuru Funato, and Yoichi Kawakami

Citation: Journal of Applied Physics 113, 183523 (2013); doi: 10.1063/1.4804328

View online: http://dx.doi.org/10.1063/1.4804328

View Table of Contents: http://scitation.aip.org/content/aip/journal/jap/113/18?ver=pdfcov

Published by the AIP Publishing

\section{Articles you may be interested in}

Dislocation blocking by AIGaN hot electron injecting layer in the epitaxial growth of GaN terahertz Gunn diode J. Appl. Phys. 114, 104508 (2013); 10.1063/1.4820460

Distorted wurtzite unit cells: Determination of lattice parameters of nonpolar a -plane AIGaN and estimation of solid phase Al content

J. Appl. Phys. 109, 013107 (2011); 10.1063/1.3525602

Heteroepitaxy of AIGaN on bulk AIN substrates for deep ultraviolet light emitting diodes

Appl. Phys. Lett. 91, 051116 (2007); 10.1063/1.2766841

Origins of threading dislocations in GaN epitaxial layers grown on sapphire by metalorganic chemical vapor deposition

Appl. Phys. Lett. 78, 1544 (2001); 10.1063/1.1352699

Effect of Si doping of metalorganic chemical vapor deposition-GaN templates on the defect arrangement in hydride vapor phase epitaxy-GaN overgrown layers J. Appl. Phys. 88, 5729 (2000); 10.1063/1.1318366

\section{$\underset{\substack{\text { Aubloning } \\ \text { Al }}}{A}$ Re-register for Table of Content Alerts}




\title{
Heteroepitaxy between wurtzite and corundum materials
}

\author{
Yuki Hayashi, Ryan G. Banal, Mitsuru Funato, and Yoichi Kawakami \\ Department of Electronic Science and Engineering, Kyoto University, Kyoto 615-8510, Japan
}

(Received 25 February 2013; accepted 24 April 2013; published online 13 May 2013)

\begin{abstract}
Heteroepitaxy of wurtzite semiconductors on corundum substrates is widely used in modern optoelectronic devices, because both crystals belong to the same hexagonal close-packed system. However, the constituent atoms in the wurtzite structure align in an ideal hexagon within the (0001) plane, whereas those in the corundum structure are displaced due to empty octahedral sites. Herein, we demonstrate that this atomic arrangement mismatch at the interface generates low-angle grain boundaries in epilayers, and step bunching of corundum substrates with an even number of molecular layers can eliminate the boundaries. Furthermore, we propose that the weakened epitaxial relationship between epilayers and substrates also eliminates low-angle grain boundaries, which may be useful for practical applications. (C) 2013 AIP Publishing LLC. [http://dx.doi.org/10.1063/1.4804328]
\end{abstract}

\section{INTRODUCTION}

Heteroepitaxy is of great technical importance for materials on foreign substrates because it can be used to expand available systems and lead to innovative applications. Typical examples of heteroepitaxial materials include GaAs on Si (Refs. 1 and 2) and GaN on sapphire ${ }^{3,4}$ or Si. $^{5,6}$ A critical factor that governs heteroepitaxy is the dissimilarity between the epilayer and substrate. Due to a lattice mismatch, epilayers grow pseudomorphically up to a critical thickness, ${ }^{7}$ at which misfit dislocations are induced to reduce the strain energy in the system. Domain matching epitaxy, which grows strain-free films, while confining misfit dislocations near the interface, has been proposed for obtaining high-quality films. ${ }^{8}$ Meanwhile, crystallographic mismatch often leads to rotation domains. General criteria to describe the rotation degree have already been proposed. ${ }^{9,10}$ Previous studies, however, have been based on the translational (lattice) mismatch and rotational mismatch of the primitive lattices, and have not focused on the interfacial atomic arrangement.

In this study, we demonstrate that the interfacial atomic arrangement affects the epilayer crystalline properties, using a technologically important heterostructure: wurtzite IIInitride semiconductors on corundum sapphire (0001). InGaN quantum wells (QWs) on sapphire (0001) substrates have produced visible light emitters, ${ }^{4}$ and furthermore, due to potential applications in ultraviolet emitters and power electronic devices, AlN-based heterostructures on sapphire have recently received much attention. ${ }^{11-13}$

Both III-nitrides and II-oxides on sapphire structures experience significant mismatch, in lattice parameters as well as crystallographic structures. To minimize lattice mismatch, a $30^{\circ}$ rotation occurs within the $(0001)$ heterointerface. ${ }^{4,14}$ Additionally, in-plane rotation domains with a small angle difference of $3^{\circ}-5^{\circ}$ have been reported for GaN (Ref. 15) and AlN. ${ }^{16-18}$ Such high-angle and low-angle rotation domains necessarily introduce grain boundaries, bordered by edge dislocation arrays, and have detrimental consequences for epilayer and device properties. In this work using AlN-onsapphire (0001) heterostructures, we demonstrate that the mismatch in the interface atomic arrangements between wurtzite and corundum crystals causes low-angle grain boundaries in the epilayer, but sapphire substrate steps with an even number of molecular layers can eliminate the boundaries.

\section{AIN SAMPLES}

AlN layers with a thickness of $\sim 600 \mathrm{~nm}$ were grown directly on sapphire (0001) substrates by metalorganic vapor phase epitaxy (MOVPE), typically at $1200{ }^{\circ} \mathrm{C} .{ }^{19}$ The superior quality of the grown AlN layers is demonstrated by AlGaN QWs on AlN layers with extremely high emission efficiencies. $^{12,13}$ In addition, many samples were grown under different conditions, including continuous and alternating source supply methods. ${ }^{19,20}$ Their X-ray diffraction (XRD) line widths were in the range of 30-300 arc sec for the (0002) symmetric diffraction, and 250-1450 arc sec for (1012) asymmetric diffraction, which correspond to densities of $2 \times 10^{6}-2 \times 10^{8} / \mathrm{cm}^{2}$ for screw dislocations and $7 \times 10^{8}-3 \times 10^{10} / \mathrm{cm}^{2}$ for edge dislocations.

\section{FORMATION OF LOW-ANGLE GRAIN BOUNDARIES}

\section{A. Experimental results}

Prior to epitaxy, the sapphire substrates were thermally annealed in $\mathrm{H}_{2}$ at $1220^{\circ} \mathrm{C}$. Figure 1 shows the atomic force microscope (AFM) images of (a) an annealed sapphire substrate and (b) AlN after epitaxy. The sapphire substrates have an unintentional off-angle of $0.06^{\circ}$, creating atomically flat terraces with widths of a few hundred $\mathrm{nm}$ and single monolayer (ML) steps. The step-terrace structure of the substrate is well replicated in the AIN epilayer, as demonstrated in Fig. 1(b).

Figure 2 shows the structural properties of the AlN epilayer assessed by transmission electron microscopy (TEM) and XRD. Cross-sectional TEM (XTEM) observations indicate that most of the threading dislocations are pure $a$-type edge dislocations. Figure 2(a) is a plane-view TEM brightfield image, where not only isolated edge dislocations but also line structures denoted by arrows can be observed. To 

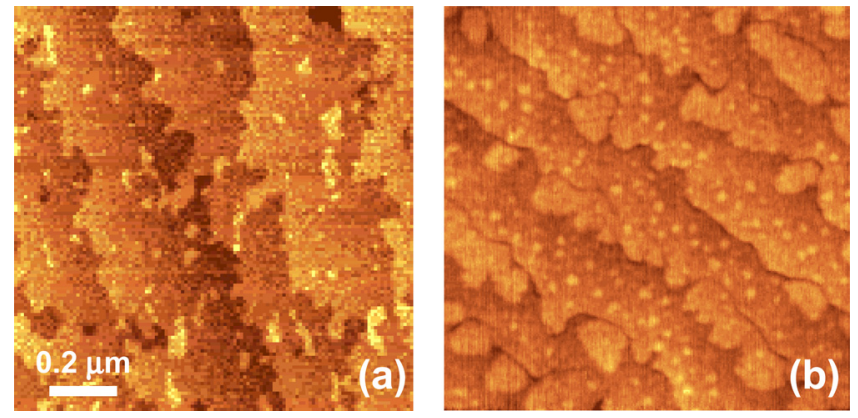

FIG. 1. AFM images of (a) a sapphire (0001) substrate and (b) an AlN epilayer. identify the origin of the line structure, Fig. 2(b) displays a high-resolution image; line structures are clearly edge dislocation arrays with an estimated distance $D$ between dislocations of $\sim 4 \mathrm{~nm}$. Moreover, the lattice image in Fig. 2(b) indicates that on each side of the dislocation array, the crystals rotate by $\psi=4^{\circ}-5^{\circ}$ around the [0001] axis, suggesting the formation of a low-angle grain boundary. If this is the case, $D$ should equal $b / \psi$, where $b$ is the lattice transition vector (i.e., Burgers vector). ${ }^{21}$ Substituting the observed $D=4 \mathrm{~nm}$ and $\psi=4^{\circ}-5^{\circ}$, we obtain $b=0.28-0.35 \mathrm{~nm}$, which includes $b$ for edge dislocations in AIN $(0.3112 \mathrm{~nm})$. Hence, we conclude that dislocation arrays are composed of edge dislocations located along grain boundaries.
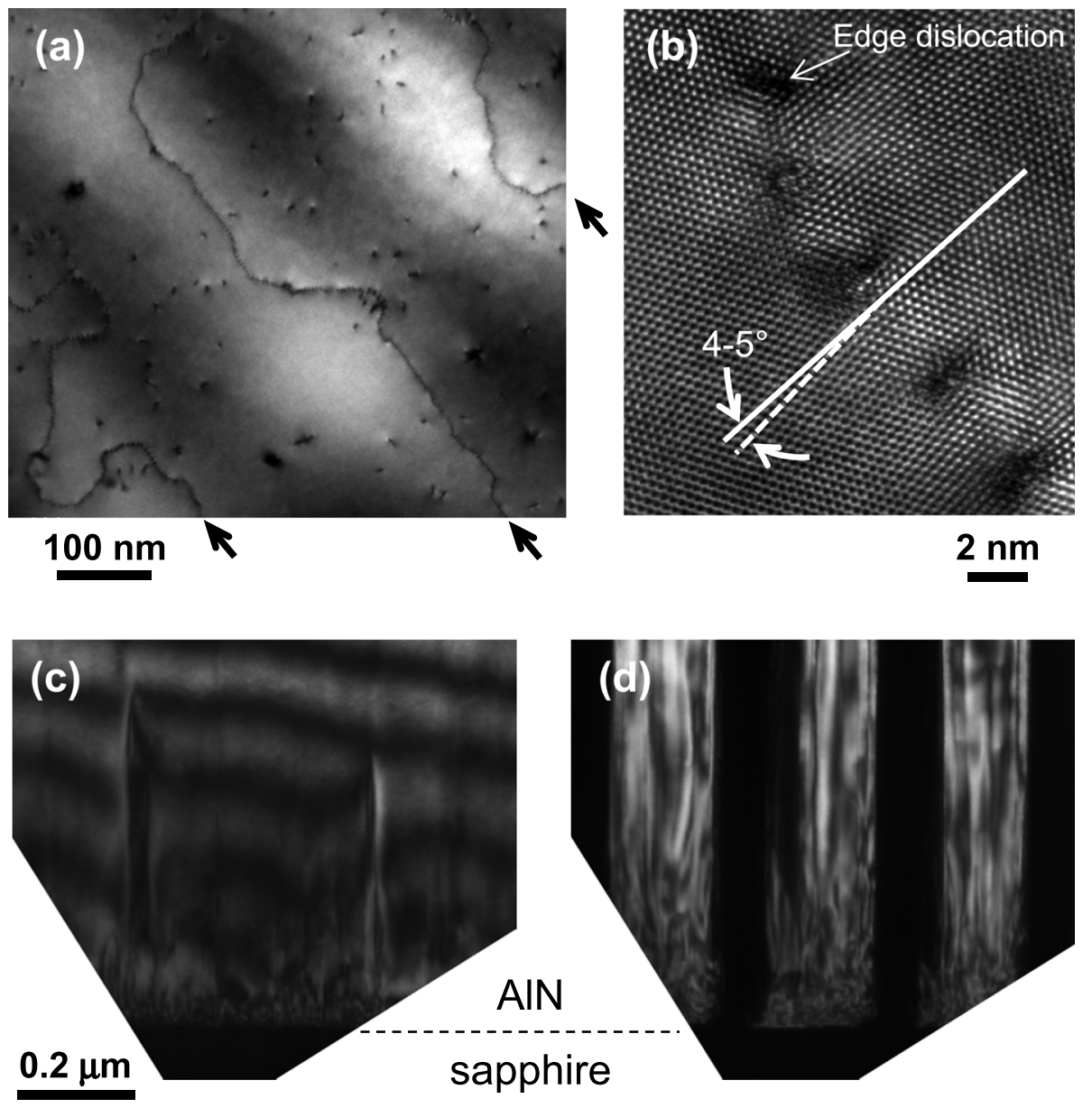

FIG. 2. Plan-view (a) bright field and (b) highresolution TEM images of AIN. XTEM dark field images of AlN viewed along the [1100] direction under (c) $g=[0002]$ and (d) $[11 \overline{2} 0]$ two-beam conditions. (e) XRD $\phi$ scans of asymmetric planes in AlN and sapphire substrate.

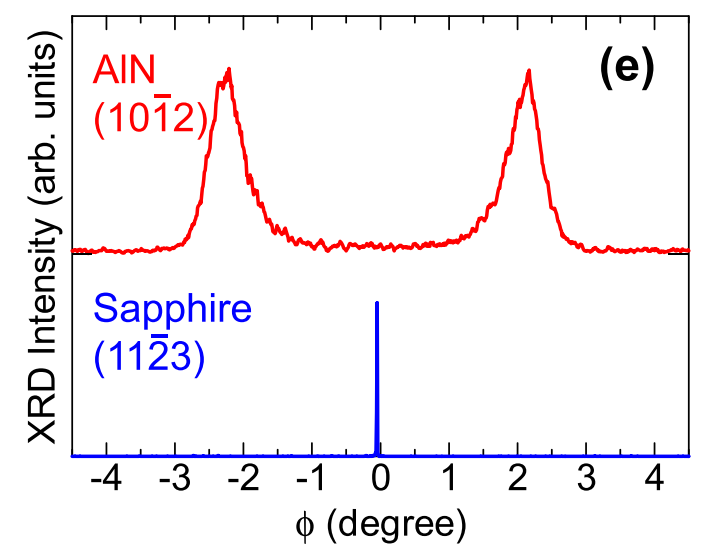


Figures 2(c) and 2(d) are (1100) XTEM dark field images taken at the same position under (c) $g=[0002]$ and (d) [11 $\overline{2} 0]$ two-beam conditions. With $\boldsymbol{g}=$ [0002], which is an invisibility condition for edge dislocations, the image is nearly contrast-free, whereas $g=[11 \overline{2} 0]$ creates striking contrast. These findings indicate that the contrast is caused by columnar two phases well aligned along the [0001] direction, but misaligned within the (0001) plane, consistent with Fig. 2(b). Furthermore, the width of the contrast in Fig. 2(d) is comparable to the spacing of the line structure in Fig. 2(a). Therefore, it is reasonable to consider that the contrast in the XTEM is derived from grains with low-angle boundaries. Another important conclusion from the XTEM is that the grain structure is generated at the initial stage of the growth and is inherited during the growth. The sapphire-AlN interface seems to trigger the grain formation.

In addition to the nanoscopic TEM observations, macroscopic assessments were conducted by XRD. The $\omega$ scan of the (0002) symmetric diffraction exhibits a line width as narrow as 65 arc sec, indicating highly $c$-oriented growth. On the other hand, the AlN (1012) asymmetric diffraction in the $\phi$ scan is divided into two components peaking at $\pm 2.2^{\circ}$ with respect to the sapphire (1123) diffraction [Fig. 2(e)]. If AlN is a perfect single-phase crystal, the AlN (1012) diffraction should be a single peak at around the same angular position as the sapphire (1123) diffraction. [Note that AlN is rotated $30^{\circ}$ in the (0001) plane.] Therefore, Fig. 2(e) indicates the presence of two distinct phases in the AlN epilayer. Because the estimated XRD peak separation of $4.4^{\circ}$ agrees well with the in-plane misorientation between two adjacent grains revealed by TEM, the nanoscopic observation of the grain boundary is not due to chance, but due to grains with regular rotation angles formed in the entire area of the epilayer. Grains seem to be formed intrinsically.

\section{B. The model}

Because of Fig. 2(d) and the additional experimental result showing that $\mathrm{AlN}$ on $\mathrm{SiC}$ substrates does not produce such a low-angle rotation (data not shown), we focus on the sapphire-AlN interface. The distance between the dislocation arrays in AlN (Fig. 2) coincides well with the terrace widths of the sapphire substrate and AlN (Fig. 1). Therefore, we deduce that the step-terrace structure on sapphire is responsible for the low-angle grain boundaries. Sapphire $\left(\mathrm{Al}_{2} \mathrm{O}_{3}\right)$ has a corundum structure, where the $\mathrm{O}$ atoms are displaced by $\sim 0.02 \mathrm{~nm}$ within the (0001) plane to align in a distorted hexagon, due to the empty cation sites [Fig. 3(a)]. Depending on the position of the $\mathrm{Al}$ empty sites, two differently distorted hexagons appear alternately along the $c$ axis, with a period of ML; as illustrated in Figs. 3(a) and 3(b), we define these two molecular layers as A and B stacking. It is interesting to note that if the distorted hexagons are divided into three tetragonal parts, 1, 2, and 3, as defined in Fig. 3(b), 1, 2, and 3 show a clockwise sequence for A stacking, and a counterclockwise sequence for B stacking. We presume that AlN on the Astacking sapphire and that on the B-stacking sapphire rotate in the opposite direction due to the opposite rotational geometry of the distorted hexagons. When 1-ML steps and terraces are structured on the sapphire (0001) surface, A and B stacking should appear side by side, as shown in Fig. 3(c), which may cause the low-angle grain boundaries.

\section{Interface potential calculation}

To prove the above hypothesis, we initially simulated the rotation angle using a model based on the Tersoff potential. ${ }^{22}$ Presently, the detailed interface structure has yet to be clarified, either experimentally or theoretically. In particular, the parameter describing the preferred angle between $\mathrm{O}-\mathrm{Al}$ and $\mathrm{Al}-\mathrm{N}$ bonds comprising the interfacial O-Al-N structure is unknown, preventing detailed analyses that fully consider lattice distortion. Therefore, the model is simplified, so that the $\mathrm{O}$ in sapphire and the $\mathrm{Al}$ in AlN form the sapphire-AlN interface without distorting the sapphire and AlN lattices. We then consider the rotation, as well as the in-plane and out-of-plane translations of the six $\mathrm{Al}$ atoms aligned in an unstrained AlN lattice with an Al-Al spacing of $0.3112 \mathrm{~nm}$ around the $\mathrm{Al}$ sites of sapphire, as illustrated in Fig. 4(a). These assumptions minimize the potential energies in the bulk regions of sapphire and AlN, and only the potential energy with respect to the interface Al-O bonds must be minimized.

The potential $U$ is defined as $U=\sum_{i j}\left\{A \exp \left(-\lambda_{1} r_{i j}\right)\right.$ $\left.-B \exp \left(-\lambda_{2} r_{i j}\right)\right\}$, where the first and second terms are related to the repulsive and attractive energies between $\mathrm{O}$ and $\mathrm{Al}$, (a)

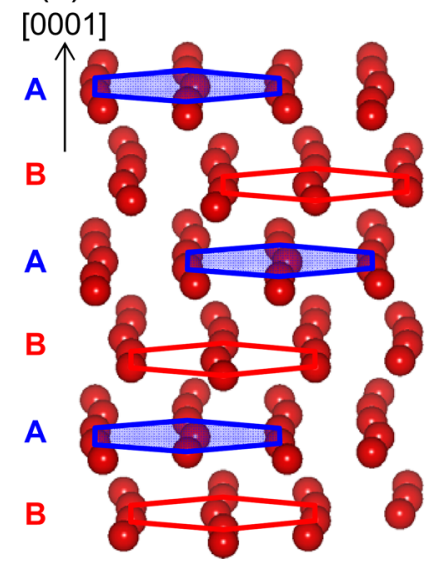

(b)

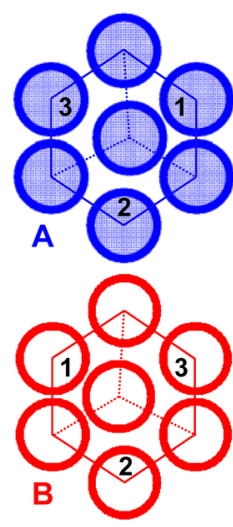

(c)

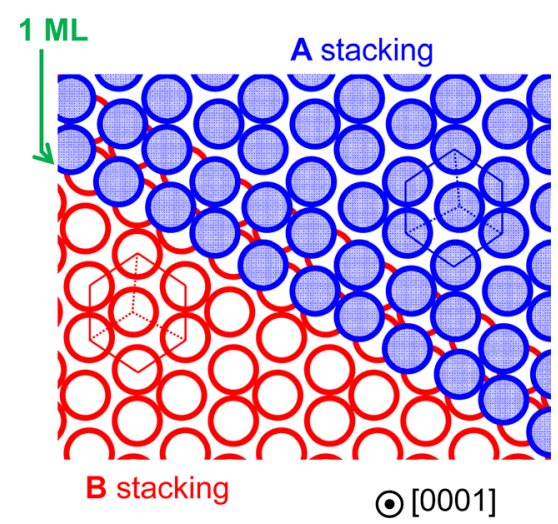

FIG. 3. Schematic structure of sapphire: (a) position of the $\mathrm{O}$ atoms, (b) top view of the $\mathrm{O}$ atoms in $\mathrm{A}$ and $\mathrm{B}$ stacking, and (c) step-terrace structure on a sapphire (0001) surface with respect to the $\mathrm{O}$ atoms. 

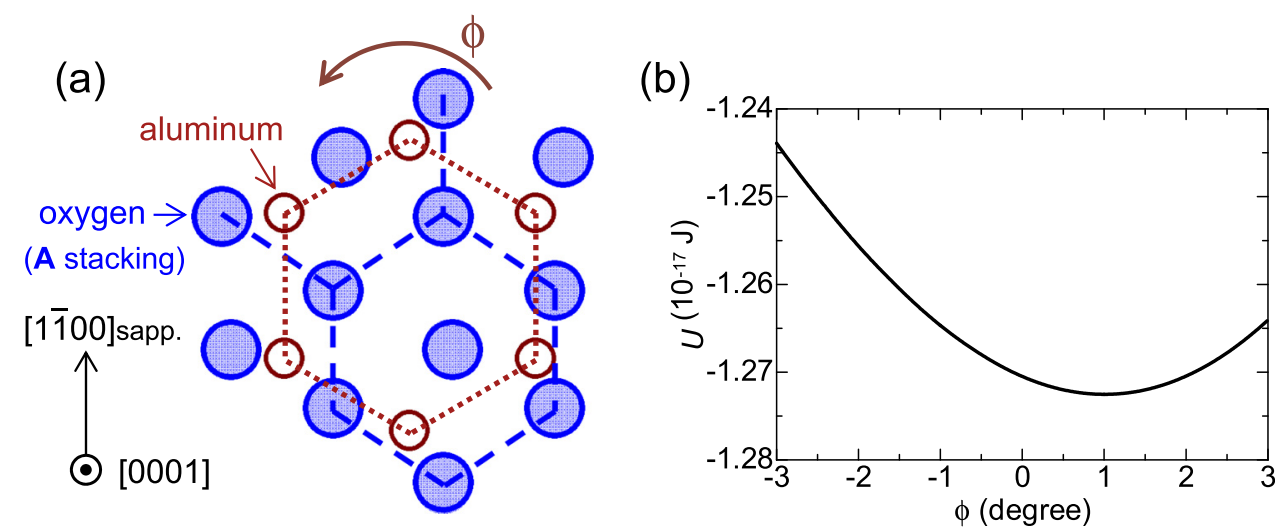

FIG. 4. (a) AlN growth model on a sapphire substrate. (b) AlN rotation angle dependence of potential $U$.

respectively, and $r_{i j}$ denotes for the Al-O distance. For the repulsive energy, $A=4.861 \times 10^{-16} \mathrm{~J}$ and $\lambda_{1}=0.3734 / \mathrm{nm}$ have been reported, ${ }^{23}$ while $B$ and $\lambda_{2}$ for the attractive energy were determined by fitting $A \exp \left(-\lambda_{1} r_{i j}\right)-B \exp \left(-\lambda_{2} r_{i j}\right)$ to the Al-O bond energy in sapphire, when $r_{i j}$ is equal to the Al$\mathrm{O}$ distance in sapphire $(0.1852 \mathrm{~nm})$. This procedure gives $B=1.745 \times 10^{-17} \mathrm{~J}$ and $\lambda_{2}=0.1412 / \mathrm{nm}$. Figure $4(\mathrm{~b})$ is the calculated result as a function of the rotation angle $\phi$, where

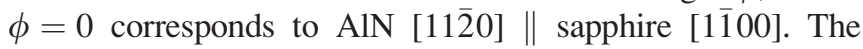
potential is minimized when $\phi$ is $1.0^{\circ}$. Because $\mathrm{AlN}$ on A and B stackings should rotate in opposite directions, due to the opposite rotational geometry of the $\mathrm{O}$ alignments, adjacent grains may rotate a total of $2.0^{\circ}$. This prediction qualitatively agrees with the experimental results discussed in Fig. 2.

It is plausible that the transition of the $\mathrm{Al}$ coordination from six in sapphire to four in AlN, which is left out of the above simulation, plays a role in determining the crystallographic properties of the epilayer. However, as demonstrated experimentally below, the interface geometry plays a much greater role. [One supporting factor is that when $c$-oriented AlN is grown on $(11 \overline{2} 0)$ sapphire, low-angle grain boundaries do not appear.]

\section{PROVING THE MODEL}

\section{A. Step bunching of sapphire with 2-ML step height}

If two oppositely distorted hexagons with A and B stackings are the origin of low-angle grain boundaries, then growing AlN on a sapphire surface with either A or B stacking should eliminate these boundaries. As schematically illustrated in Fig. 5, such sapphire surfaces may be realized by step bunching with an even number of ML steps. Although step bunching has been reported to occur on sapphire vicinal surfaces upon annealing in air, ${ }^{24-26}$ we need to establish a condition in which even-numbered ML steps are stably realized. Thus, we annealed sapphire with an off-angle of $0.5^{\circ}$, $1^{\circ}$, or $3^{\circ}$ along the [1120] or [1 100$]$ direction in air at $1150^{\circ} \mathrm{C}$. When the incline is along the [1100] direction, step edges occasionally misalign by $30^{\circ}$ from the initial (1100) step and coalesce to form step junctions. On the other hand, the incline along the [1120] direction leads to very straight steps, similar to Ref. 26. Hereafter, we concentrate on sapphire inclined along the [112̄0] direction, because straight steps may offer highly uniform step-terrace structures with even-numbered ML steps.

Figure 6 shows the relation between the sapphire offangle $\theta$ and formed terrace widths $L$, where the symbols and broken curves denote experimental data and calculated results using $L=\mathrm{ML} / \tan \theta$, respectively. The terrace width was assessed at ten random points from the AFM images. For $0.5^{\circ}$ and $3^{\circ}$ off sapphire, the annealing time was $45 \mathrm{~min}$, while for $1^{\circ}$ off sapphire, it was 30, 45, or $60 \mathrm{~min}$. Figure 6 shows that the terrace width is about $25 \mathrm{~nm}$ and is independent of both the off-angle and annealing time. This can be interpreted by considering step bunching as a consequence of reconstructing evaporated atoms on the surface. The terrace width is determined by the diffusion length of migrating atoms, which depends on the annealing temperature but not on the off-angle or annealing time. When the off-angle is $1^{\circ}$ and terrace width is $\sim 25 \mathrm{~nm}$, the step height should be $2 \mathrm{ML}$, as demonstrated by the broken curve in Fig. 6. Furthermore, it is noteworthy that for the $1^{\circ}$ off sapphire, annealing at an elevated temperature of $1200^{\circ} \mathrm{C}$ also provides 2-ML steps and $\sim 25 \mathrm{~nm}$ wide terraces, despite the enhanced migration. This observation is reasonable because diffusion of $\sim 40 \mathrm{~nm}$ is necessary to realize further step bunching with a height of 3 ML (Fig. 6), which cannot be achieved by a small increase of $50^{\circ} \mathrm{C}$ in the annealing temperature. Thus, we

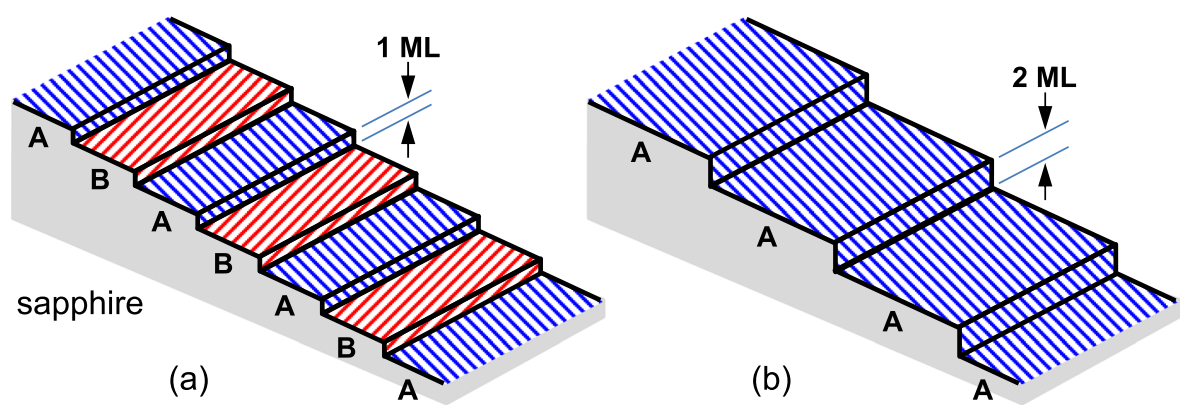

FIG. 5. Step-terrace structures of sapphire with (a) 1-ML and (b) 2-ML steps. 


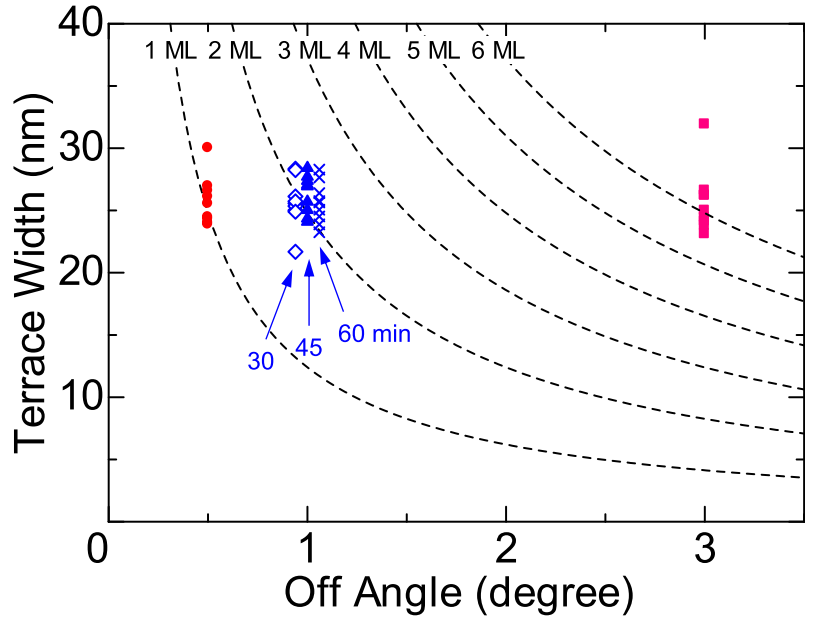

FIG. 6. Sapphire off-angle, $\theta$, dependence of the terrace width, $L$, on the sapphire surface annealed at $1150^{\circ} \mathrm{C}$ in air. The curves are the calculated $L$ based on $L=\mathrm{ML} / \tan \theta$, using the step height as a parameter.

have established conditions (annealing at $\sim 1150^{\circ} \mathrm{C}$ using $1^{\circ}$ off sapphire) to stably provide 2-ML step bunching.

Homogeneity of the step-terrace structure formed on the $1^{\circ}$ off sapphire annealed at $1150{ }^{\circ} \mathrm{C}$ for 60 min was examined. Figure 7(a) displays an AFM image with regular steps. To quantify the observation, Fig. 7(b) shows its fast Fourier transform (FFT) image. Two bright spots are detected at positions corresponding to a terrace width of $\sim 25 \mathrm{~nm}$, which in turn, corresponds to 2-ML steps, as calculated in Fig. 6. Hence, a high degree of homogeneity is confirmed.

\section{B. AIN growth on step-bunched sapphire}

The AlN layers were grown on sapphire substrates with 2-ML step bunching under the same conditions as the AlN shown in Figs. 1 and 2. The XRD $\phi$ scan [Fig. 8(a)] shows that an AlN single peak appears at $\sim 0.8^{\circ}$ with respect to the sapphire peak, indicating that although AlN slightly rotates in the plane, the rotating direction is unified by using sapphire covered with a single stacking of either A or B. It is interesting to note that the rotation angle of $\sim 0.8^{\circ}$ is close to the prediction [Fig. 4(b)], implying that the formation of the lowangle grain boundary may enlarge the rotation angle of the AlN epilayer to $\pm 2.2^{\circ}$. Furthermore, the plan-view TEM bright field image [Fig. 8(b)] confirms that edge dislocation arrays are completely eliminated, indicating the absence of
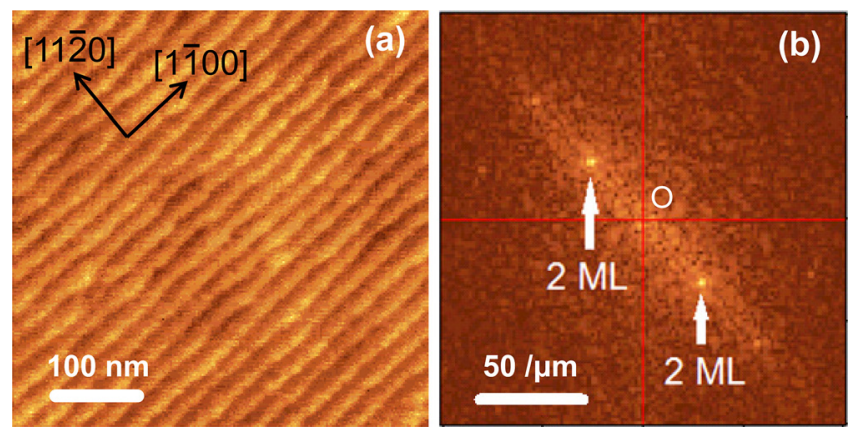

FIG. 7. (a) AFM image of $1^{\circ}$ off sapphire (0001) substrate annealed at $1150^{\circ} \mathrm{C}$ in air. (b) FFT image of (a). low-angle grain boundaries. It is also noteworthy that the density of isolated dislocations is nearly the same as that in AlN with low-angle grain boundaries. Therefore, our method successfully eliminates grains without degrading the film quality on terraces. These observations clearly demonstrate our hypothesis that the mismatch in the interface atomic arrangements between wurtzite AIN and corundum sapphire intrinsically causes grains with the opposite in-plane rotation directions.

\section{Discussion}

Thus far, only the direct growth of AlN on sapphire has been described, because high-quality AlN can be achieved even without a nucleation layer, such as a low-temperature buffer layer. ${ }^{19,20,27}$ However, to see the effect of initial nucleation processes, we also have examined $\sim 20$-nm-thick AlN low-temperature $\left(600\right.$ or $\left.1000^{\circ} \mathrm{C}\right)$ buffer layers and nitridation of the sapphire substrates. It has been confirmed that as long as the initial sapphire surfaces are sufficiently annealed in $\mathrm{H}_{2}$, which is quite important process as demonstrated in Sec. V, all the samples reproduce the phenomenon discussed in this study. Furthermore, two different MOVPE machines with vertical or horizontal reactors have exhibited consistent results. In addition, structures similar to our observations have been reported for GaN (Ref. 15) and AlN. ${ }^{16-18,27}$ Particularly for AlN, the growth method is not only MOVPE but also solid-phase epitaxy. ${ }^{18}$ On the other hand, it has been reported that $\mathrm{Al}$ preflow ${ }^{16}$ or a roughened surface $^{18}$ may eliminate low-angle grain boundaries in AlN. For GaN/sapphire, low temperature buffer layers adopted to compensate for the low wettability between $\mathrm{GaN}$ and sapphire ${ }^{4}$ have high mosaicity and may be an obstacle to explicitly observing low-angle grain boundaries related to interfacial geometry. All these findings have led us to conclude that the formation of the specific grain structure is an intrinsic property for wurtzite materials grown on corundum materials with the (0001) surface, and should be observed unless some extrinsic factors weaken the epitaxial relationship.

Considering the origin, we deduce that similar phenomena possibly take place when a crystal with rotational symmetry is heteroexpitaxially grown on the sapphire (0001) plane. Besides III-nitride and II-oxide semiconductors, growths of Si (Refs. 28 and 29), SiC (Ref. 30), Cu (Ref. 31), $\mathrm{Nb}$ (Ref. 32), and even graphene ${ }^{33}$ on sapphire have already been demonstrated experimentally, and those material systems may experience the same issue. For corundum substrates, our proposal will offer a simple way to eliminate low-angle grain boundaries with edge dislocations and promote the development of novel devices.

\section{INTERFACE CONTROL TO ELIMINATE LOW-ANGLE GRAIN BOUNDARIES IN AIN ON SAPPHIRE (0001) ON-AXIS SUBSTRATES}

In the preceding sections, we have proposed the model that explains formation mechanism of low-angle grain boundaries in AlN, and proven it, using sapphire (0001) vicinal substrates. In practice, however, sapphire (0001) nearly 

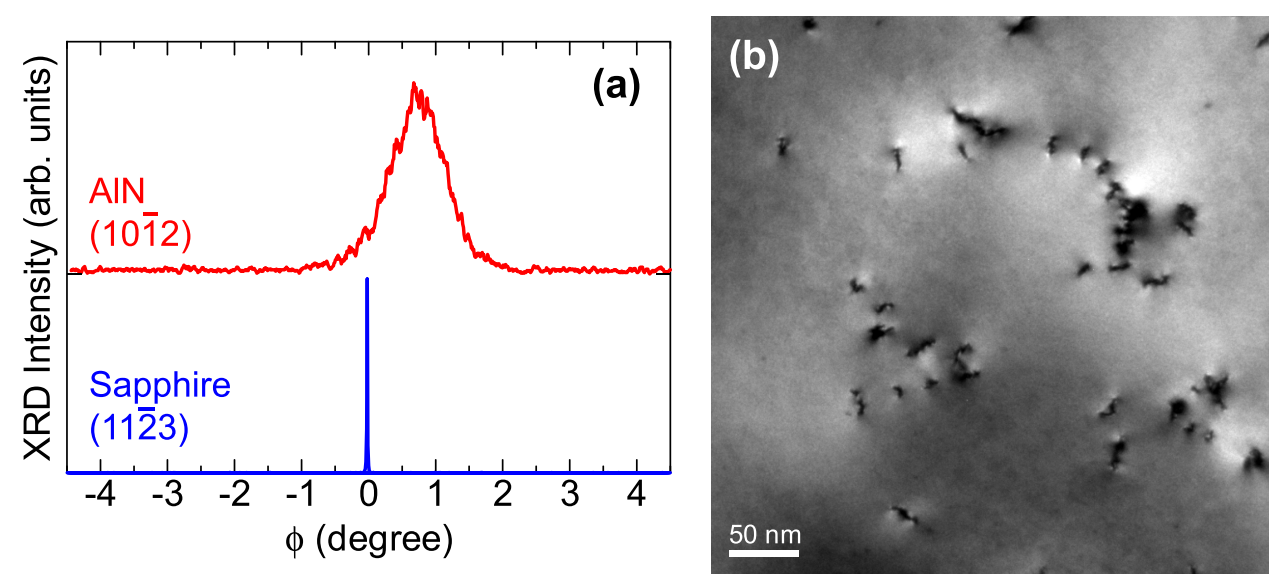

FIG. 8. (a) XRD $\phi$ scan of AlN on the $1^{\circ}$ off sapphire (0001) substrate annealed at $1150^{\circ} \mathrm{C}$. (b) Plan-view TEM bright field image of AlN on the sapphire substrate.

on-axis substrates are widely used. In this section, we demonstrate that control of the interfacial bonding can eliminate low-angle grain boundaries in AlN even on the (0001) on-axis sapphire substrates.

\section{A. Control of the surface termination of sapphire}

Sapphire (0001) nominally singular substrates were first annealed in air under the conditions established in Sec. IV A. At this stage, the sapphire surface was quite stable and unreactive, since experiments showed that without additional high-temperature annealing in $\mathrm{H}_{2}$ atmosphere, $\mathrm{GaN}$ cannot nucleate on this surface at all, even at the typical growth temperature for low-temperature buffer layers $\left(\sim 600^{\circ} \mathrm{C}\right)$. This is why in the preceding sections, the sapphire surface was subsequently annealed in $\mathrm{H}_{2}$ atmosphere at high temperatures.

We compare the surface states of sapphire annealed in air and that followed by annealing in $\mathrm{H}_{2}$ at $1220^{\circ} \mathrm{C}$ via Fourier transform infrared spectroscopy (FTIR). An attenuated total reflection (ATR) configuration was adopted, using a Ge crystal. The resolution was $4 \mathrm{~cm}^{-1}$. Figure 9 shows the result. After $\mathrm{H}_{2}$ annealing (process $a$ ), a signal appeared at $1730 \mathrm{~cm}^{-1}$, and no signal was detected between 3000 and $3800 \mathrm{~cm}^{-1}$. Because the hydrogen stretching frequency for $\mathrm{Al}-\mathrm{H}$ is in the range of $1700-1800 \mathrm{~cm}^{-1}$, while that for $\mathrm{O}-\mathrm{H}$ should appear in the $3000-3800 \mathrm{~cm}^{-1}$ range, ${ }^{34}$ the observed peak can be attributed to the surface termination with $\mathrm{Al}-\mathrm{H}$

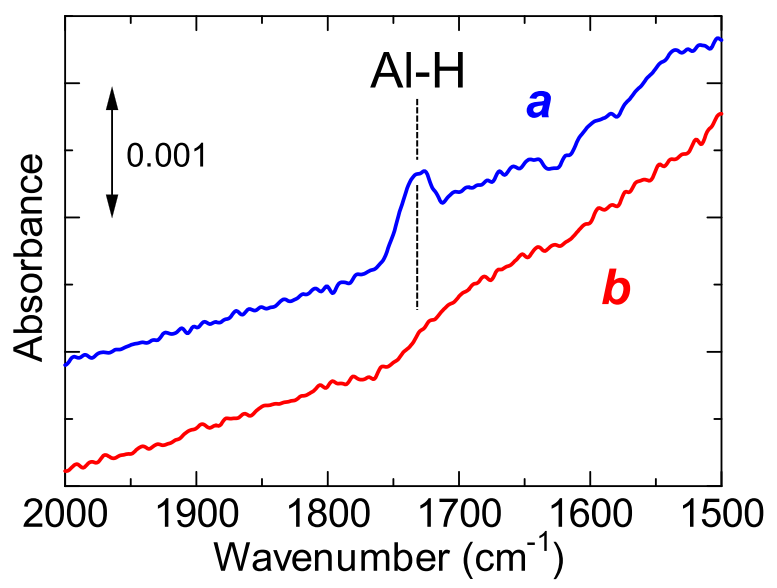

FIG. 9. FTIR spectra for sapphire with (process $a$ ) and without (process $b$ ) annealing in $\mathrm{H}_{2}$ at $1220^{\circ} \mathrm{C}$. bonds. On the other hand, the sapphire surface just after air annealing (process $b$ ) shows no FTIR signals within the spectral range of interest.

AlN films were subsequently grown on these surfaces. Figure 10 shows their temperature profiles. Prior to the processes shown in Fig. 10, all sapphire substrates were subjected to air annealing at $1150^{\circ} \mathrm{C}$. In profile $a$, the air-annealed sapphire is exposed to $\mathrm{H}_{2}$ at $1220^{\circ} \mathrm{C}$ for $10 \mathrm{~min}$, whereas in profile $b$, the substrate temperature is kept relatively low at $1000{ }^{\circ} \mathrm{C}$. Therefore, the sapphire surface just before the AlN growth is expected to be terminated with $\mathrm{H}$ atoms in profile $a$, but not in profile $b$. (Data supporting this hypothesis are given below.)

On these surfaces, medium-temperature AlN buffer layers were grown at $1000^{\circ} \mathrm{C}$. The aim of the buffer layer in profile $b$ is to preserve the stable sapphire surface until the AIN growth is initiated, whereas that in profile $a$ is to grow AlN under conditions identical to profile $b$, except for the initial surface states of sapphire. After growing mediumtemperature AlN buffer layers for $1.5 \mathrm{~min}$, which corresponds to a thickness of $45 \mathrm{~nm}$, AlN was grown at the optimal temperature of $1200{ }^{\circ} \mathrm{C}$.

Figure 11 shows XRD $\phi$ scans of AlN grown on the sapphire (0001) on-axis substrates with different temperature profiles $a$ and $b$. The diffractions of the asymmetric AlN

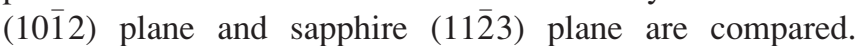
Profile $a$ provides a similar XRD profile to Fig. 2(e), indicating the formation of low-angle grain boundaries, whereas profile $b$ eliminates the grains with low-angle boundaries.

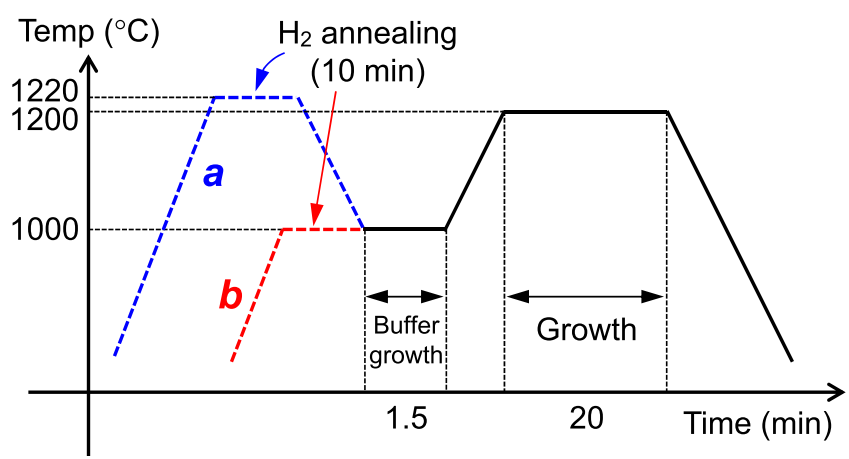

FIG. 10. Two temperature profiles for AIN growth with the control of sapphire surface termination. Profiles $a$ and $b$ correspond to processes $a$ and $b$ for FTIR, respectively. 


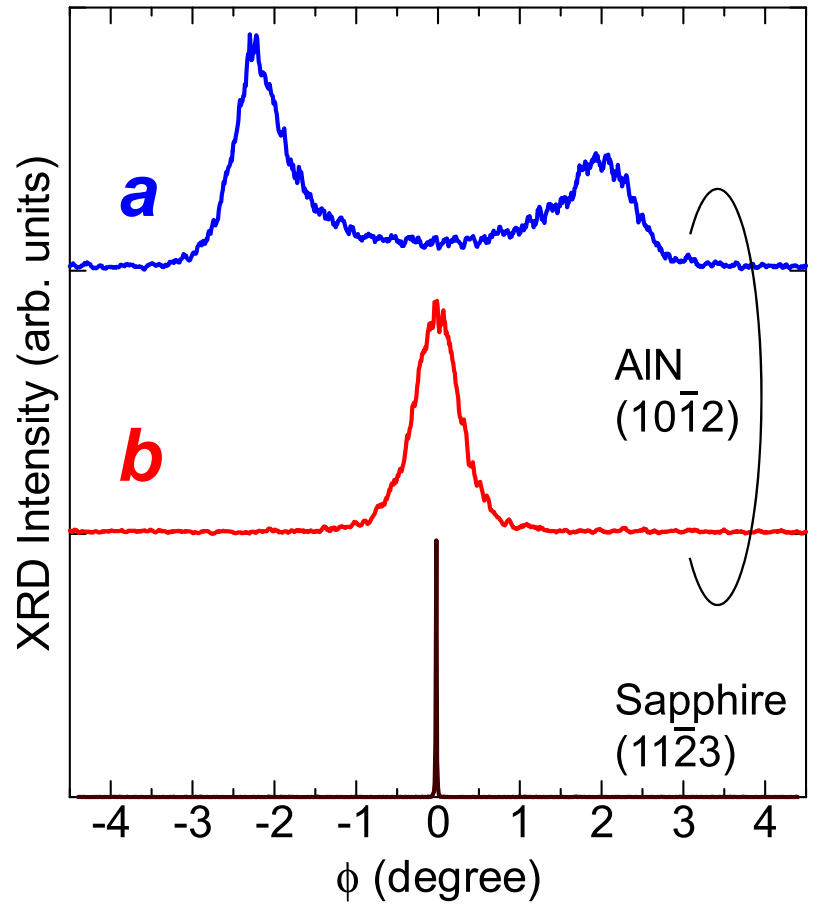

FIG. 11. XRD $\phi$ scans of AIN grown on the sapphire (0001) on-axis substrates with different temperature profiles $a$ and $b$.

These findings strongly suggest that it is not the AlN growth conditions but the sapphire surface state prior to the AlN growth that determines the formation of grains. We believe that the sapphire surface after air annealing is quite unreactive, as mentioned above, so that in profile $b$, the epitaxial relationship between sapphire and AlN is weakened. As discussed in Sec. IV C, such a situation may suppress a small-angle rotation.

Finally, the stability of the sapphire surface was examined. AlN layers were grown with the profile $b$ displayed in Fig. 11, but here the growth temperatures of the mediumtemperature AlN buffer layers were varied in the range between 900 and $1200^{\circ} \mathrm{C}$. (Note that when this temperature is $1200^{\circ} \mathrm{C}$, profile $b$ corresponds to profile $a$.) Figure $12 \mathrm{com}$ pares XRD $\phi$ scans of AlN (1012) asymmetric diffraction of the grown AlN layers. The buffer growth temperatures below $1050^{\circ} \mathrm{C}$ realize single-phase AlN, whereas those above $1100^{\circ} \mathrm{C}$ cause the splitting of the diffraction peak, indicating the introduction of low-angle grain boundaries. That is, the sapphire surface stabilized by air annealing is preserved even under $\mathrm{H}_{2}$ atmosphere up to $1050{ }^{\circ} \mathrm{C}$, and the $\mathrm{Al}-\mathrm{H}$ bonding starts to form above $1100^{\circ} \mathrm{C}$. These experimental findings lead us to conclude that the unreactive sapphire surfaces weaken the epitaxial relationship and effectively suppress low-angle grain boundaries. This is quite reasonable because the formation mechanism of the grain boundaries is intimately related to the interfacial geometrical atomic arrangement, as demonstrated in Secs. III and IV.

In this study, we have been using the term "weakened epitaxial relationship between the epilayer and substrate" to explain the observed phenomena. Generally, the epilayer structures are determined by the energy balance between competitive factors in the heterosystem. A typical example is lattice-mismatched heteroepitaxy; an epilayer initially grows pseudomorphically, but eventually induces misfit dislocations.

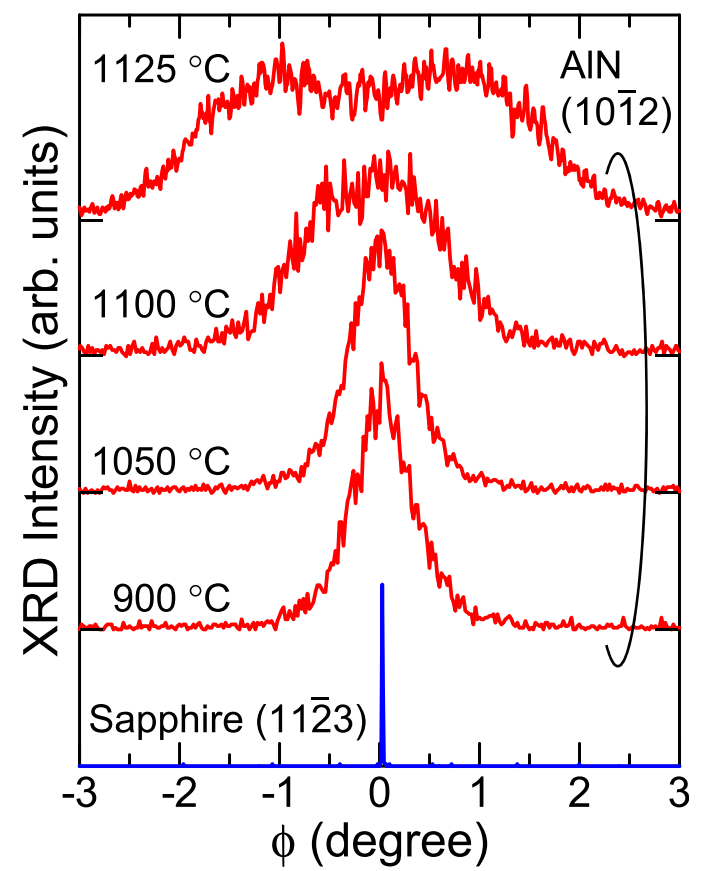

FIG. 12. XRD $\phi$ scans of AlN grown with profile $b$. The temperatures shown in the figure are those for the buffer layers, on which AlN was grown at the optimal temperature of $1200^{\circ} \mathrm{C}$ for all the samples.

This is because accommodating the misfit strain within the epilayer is energetically favorable for a thin layer, but releasing the strain by misfit dislocations is favorable for a thick layer. When a wurtzite epilayer well inherits the nature of a corundum substrate, the formation of low-angle grain boundaries could be the most stable structure, as demonstrated in Sec. III. On the other hand, when the surface of a corundum substrate is less reactive, the epilayer is relatively free from the constraints of the substrate, and could form a single phase crystal without forming low-angle grain boundaries, because breaking the epitaxial relationship is energetically more favorable than forming grains with a low-angle difference. This is the situation that we call the "weakened epitaxial relationship" and that of AlN growth on air-annealed sapphire, shown in this section. It is noteworthy that the bond strengths of diatomic molecules are in the order of Al-O $(502 \mathrm{~kJ} / \mathrm{mol}) \gg \mathrm{Al}-\mathrm{N}(368 \mathrm{~kJ} / \mathrm{mol})>\mathrm{Al}-\mathrm{H}$ $(288 \mathrm{~kJ} / \mathrm{mol}),{ }^{35}$ which could explain the less reactive sapphire surface after air annealing and the recovered reactivity after the $\mathrm{H}_{2}$ treatment.

\section{SUMMARY}

We have demonstrated that the mismatch in the interface atomic arrangements causes low-angle grain boundaries accompanied by edge dislocation arrays in wurtzite AlN epilayers grown on corundum sapphire (0001) substrates. The simulation predicts that the alignment of the $\mathrm{O}$ atoms in sapphire causes a small angle $\left(\sim 1^{\circ}\right)$ in-plane rotation of AlN, and the 1-ML step-terrace structures on the sapphire surface cause low-angle grain boundaries. The experiment demonstrates that using sapphire substrates step-bunched with the even-numbered steps can eliminate the low-angle grain boundaries, strongly supporting our hypothesis that the mismatch in the interface atomic arrangements causes grains 
with the different in-plane rotation directions. Furthermore, we propose that unreactive sapphire surface produced by air annealing may suppress the low-angle rotation.

\section{ACKNOWLEDGMENTS}

The authors acknowledge JASCO for the FTIR measurements, and K. Meiji and K. Matsuda for their support in the experiments. R.G.B. was supported by the Japan Society for the Promotion of Science (JSPS) for foreign researchers from Ministry of Education, Culture and Technology (MEXT) of Japan.

${ }^{1}$ W. I. Wang, Appl. Phys. Lett. 44, 1149 (1984).

${ }^{2}$ M. Akiyama, Y. Kawarada, and K. Kaminishi, Jpn. J. Appl. Phys., Part 2 23, L843 (1984).

${ }^{3}$ H. Amano, N. Sawaki, I. Akasaki, and Y. Toyoda, Appl. Phys. Lett. 48, 353 (1986).

${ }^{4}$ S. Nakamura, S. Pearton, and G. Fasol, The Blue Laser Diode (Springer, 2000).

${ }^{5}$ T. Lei, M. Fanciulli, R. J. Molnar, T. D. Moustakas, R. J. Graham, and J. Scanlon, Appl. Phys. Lett. 59, 944 (1991).

${ }^{6}$ A. Dadgar, J. Bläsing, A. Diez, A. Alam, M. Heuken, and A. Krost, Jpn. J. Appl. Phys., Part 2 39, L1183 (2000).

${ }^{7}$ J. W. Matthews and A. E. Blakeslee, J. Cryst. Growth 27, 118 (1974).

${ }^{8}$ J. Narayan and B. C. Larson, J. Appl. Phys. 93, 278 (2003).

${ }^{9}$ M. Grundmann, T. Böntgen, and M. Lorenz, Phys. Rev. Lett. 105, 146102 (2010).

${ }^{10}$ S. C. Erwin, C. Gao, C. Roder, J. Lähnemann, and O. Brandt, Phys. Rev. Lett. 107, 026102 (2011).

${ }^{11}$ M. Shatalov, J. Yang, W. Sun, R. Kennedy, R. Gaska, K. Liu, M. Shur, and G. Tamulaitis, J. Appl. Phys. 105, 073103 (2009).

${ }^{12}$ R. G. Banal, M. Funato, and Y. Kawakami, Appl. Phys. Lett. 99, 011902 (2011).

${ }^{13}$ T. Oto, R. G. Banal, K. Kataoka, M. Funato, and Y. Kawakami, Nature Photon. 4, 767 (2010).

${ }^{14}$ C. J. Sun, P. Kung, A. Saxler, H. Ohsato, K. Haritos, and M. Razeghi, J. Appl. Phys. 75, 3964 (1994).
${ }^{15}$ B. Heying, X. H. Wu, S. Keller, Y. Li, D. Kapolnek, B. P. Keller, S. P. DenBaars, and J. S. Speck, Appl. Phys. Lett. 68, 643 (1996).

${ }^{16}$ K. Kawaguchi and A. Kuramata, Jpn. J. Appl. Phys., Part 2 44, L1400 (2005).

${ }^{17}$ Y. Tokumoto, N. Shibata, T. Mizoguchi, M. Sugiyama, Y. Shimogaki, J. S. Yang, T. Yamamoto, and Y. Ikuhara, J. Mater. Res. 23, 2188 (2008).

${ }^{18}$ K. Ueno, J. Ohta, H. Fujioka, and H. Fukuyama, Appl. Phys. Express 4, 015501 (2011).

${ }^{19}$ R. G. Banal, M. Funato, and Y. Kawakami, J. Cryst. Growth 311, 2834 (2009).

${ }^{20}$ R. G. Banal, M. Funato, and Y. Kawakami, Appl. Phys. Lett. 92, 241905 (2008).

${ }^{21}$ F. L. Vogel, W. G. Pfann, H. E. Corey, and E. E. Thomas, Phys. Rev. 90, 489 (1953).

${ }^{22}$ J. Tersoff, Phys. Rev. B 39, 5566 (1989).

${ }^{23}$ A. A. Abrahamson, Phys. Rev. 178, 76 (1969).

${ }^{24}$ L. P. Van, O. Kurnosikov, and J. Cousty, Surf. Sci. 411, 263 (1998).

${ }^{25}$ F. Cuccureddu, S. Murphy, I. V. Shvets, M. Porcu, H. W. Zandbergen, N. S. Sidorov, and S. I. Bozhko, Surf. Sci. 604, 1294 (2010).

${ }^{26}$ Y. Shiratsuchi, M. Yamamoto, and Y. Kamada, Jpn. J. Appl. Phys., Part 1 41, 5719 (2002).

${ }^{27}$ J. Bai, T. Wang, P. J. Parbrook, K. B. Lee, and A. G. Cullis, J. Cryst. Growth 282, 290 (2005).

${ }^{28}$ C. C. Chang, J. Vac. Sci. Technol. 8, 500 (1971).

${ }^{29}$ P. Chaudhari, H. Shim, B. A. Wacaser, M. C. Reuter, C. Murray, K. B. Reuter, J. Jordan-Sweet, F. M. Ross, and S. Guha, Thin Solid Films 518, 5368 (2010)

${ }^{30}$ B. S. Sywe, Z. J. Yu, S. Burckhard, J. H. Edger, and J. Chaudhuri, J. Electrochem. Soc. 141, 510 (1994).

${ }^{31}$ G. Dehm, M. Rühle, G. Ding, and R. Raj, Philos. Mag. B 71, 1111 (1995).

${ }^{32}$ Ch. Dietrich, H.-G. Boyen, and B. Koslowski, J. Appl. Phys. 94, 1478 (2003).

${ }^{33}$ M. Fanton, J. A. Robinson, C. Puls, Y. Liu, M. J. Hollander, B. E. Weiland, M. LaBella, K. Trumbull, R. Kasarda, C. Howsare, J. Stitt, and D. W. Snyder, ACS Nano 5, 8062 (2011).

${ }^{34} \mathrm{~K}$. Nakamoto, Infrared and Raman Spectra of Inorganic and Coordination Compounds, 6th ed. (Wiley, 2009).

${ }^{35}$ W. M. Haynes (editor-in-chief), CRC Handbook of Chemistry and Physics, 93rd ed. (CRC Press, Inc., 2013). 